\title{
Protein-dependent ribozymes report molecular interactions in real time
}

\author{
Jörg S. Hartig1', S. Hani Najafi-Shoushtari¹, Imke Grüne1, Amy Yan², Andrew D. Ellington², and Michael Famulok ${ }^{1 *}$
}

\begin{abstract}
Most approaches to monitoring interactions between biological macromolecules require large amounts of material, rely upon the covalent modification of an interaction partner, or are not amenable to real-time detection. We have developed a generalizable assay system based on interactions between proteins and reporter ribozymes. The assay can be configured in a modular fashion to monitor the presence and concentration of a protein or of molecules that modulate protein function. We report two applications of the assay: screening for a small molecule that disrupts protein binding to its nucleic acid target and screening for protein-protein interactions. We screened a structurally diverse library of antibiotics for small molecules that modulate the activity of HIV-1 Revresponsive ribozymes by binding to Rev. We identified an inhibitor that subsequently inhibited HIV-1 replication in cells. A simple format switch allowed reliable monitoring of domain-specific interactions between the bloodclotting factor thrombin and its protein partners. The rapid identification of interactions between proteins or of compounds that disrupt such interactions should have substantial utility for the drug-discovery process.
\end{abstract}

Most inhibitors or modulators of biological or biochemical function are small organic molecules ${ }^{1}$. Screening compound libraries for drugs is a long and tedious process, hampered by the lack of functional assays for most targets ${ }^{2,3}$. Compounding these problems, a multitude of protein targets are now arising from genome and proteome research. Therefore, it is important to identify interaction partners for as many biological macromolecules as possible, as this information can lead to an understanding of biological function and to possible therapeutic applications. Consequently, large-scale biochemical, molecular biological, or genetic approaches for experimental detection and analysis of protein-protein interactions are being explored ${ }^{4-6}$. Even when appropriate biochemical or functional screens are available, however, the limited capacities of high-throughput screens are generally unable to generate enough lead or optimized compounds for the growing number of potential targets. Moreover, few modular assays are compatible with massively parallel screening formats; instead, the assays themselves must be tailored to each target. Therefore, it has become increasingly important to develop generalizable functional assays that can be applied independently of the nature or function of a target. We are interested in using ribozymes to develop functional assays able to report molecular interactions.

Ribozymes are naturally occurring RNA sequences with catalytic activity $^{7}$. Since their discovery 20 years ago, ribozymes have been implicated in various cellular processes that involve gene expression such as RNA splicing, RNA processing, and ribosomal peptide-bond formation. The vast majority of natural ribozymes can catalyze the trans-cleavage or trans-formation of phosphodiester bonds of RNA substrates ${ }^{8,9}$. For self-cleaving RNAs such as the hammerhead and hairpin ribozymes, the products of the cleaved substrate can dissociate from the ribozyme upon cleavage, allowing turnover for signal amplification. Prominent examples of ribozymes that have been extensively studied both on a structural and mechanistic level are the group I and group II introns, the
RNase $\mathrm{P}$, the hammerhead, hairpin, and hepatitis-delta-virus ribozymes, and many more ${ }^{10-12}$. Besides their general interest in various biological systems, ribozymes with altered substrate specificity have been used for a variety of biotechnological applications, such as the ribozyme-directed cleavage of targeted mRNA sequences for controlling gene expression ${ }^{13,14}$ and the repair of somatic mutations on the mRNA level ${ }^{15,16}$.

Recently, we developed a ribozyme-based detection scheme in which an external substrate labeled with a fluorescent molecule at its $5^{\prime}$ end and a fluorescence quencher at its $3^{\prime}$ end was cleaved, resulting in fluorescence dequenching and real-time monitoring of ribozyme activity ${ }^{17}$. Using this system, we have identified a new inhibitor of the hammerhead ribozyme from a library of small organic molecules. Most importantly, the inhibitor was able to reduce hammerhead-ribozyme activity in the nucleolus of transgenic yeast cells when added to the growth medium ${ }^{18}$. Now, by rendering ribozymes protein dependent, the specific disruption of protein-nucleic acid interactions by small organic molecules or by other proteins can also be monitored in real time.

\section{Results and discussion}

Rev-dependent reporter ribozymes. We have used a variety of strategies to make the activity of the hammerhead and the hairpin ribozyme protein dependent. In our initial design (Fig. 1), the Revbinding element (RBE) of HIV-1 was fused to helix II of the hammerhead ribozyme (Fig. 1A) to generate a Rev-responsive ribozyme $(R)$. This strategy was similar to one Breaker et al. used to generate hammerhead ribozymes dependent on small organic effectors ${ }^{19}$. Addition of the Rev protein or a peptide corresponding to the arginine-rich motif (ARM) of Rev triggered a conformational switch in the $\mathrm{RBE}^{20,21}$, ultimately rendering the ribozyme inactive so that a fluorescence resonance energy transfer (FRET)-labeled substrate remained uncleaved. In the presence of a small molecule that competed with the RBE for Rev binding, cleavage activity was restored (Fig. 1A).

${ }^{1}$ Kekulé Institut für Organische Chemie und Biochemie, University of Bonn, Gerhard-Domagk-Strasse 1, 53121 Bonn, Germany. ${ }^{2}$ Department of Chemistry and Biochemistry, University of Texas, ICMB A4800/MBB 3.448BA, 26th and Speedway, Austin, TX 78712. ${ }^{*}$ Corresponding author (m.famulok@uni-bonn.de). 
A

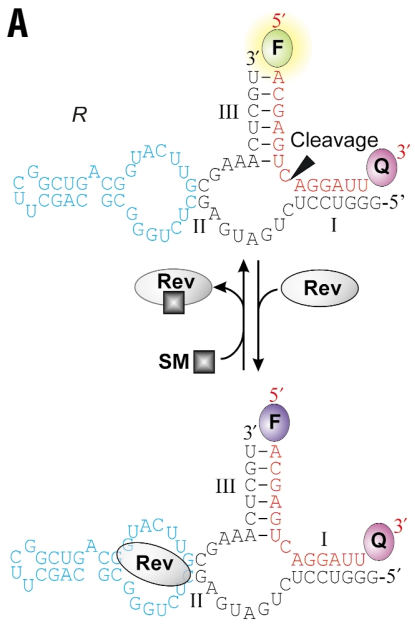

B
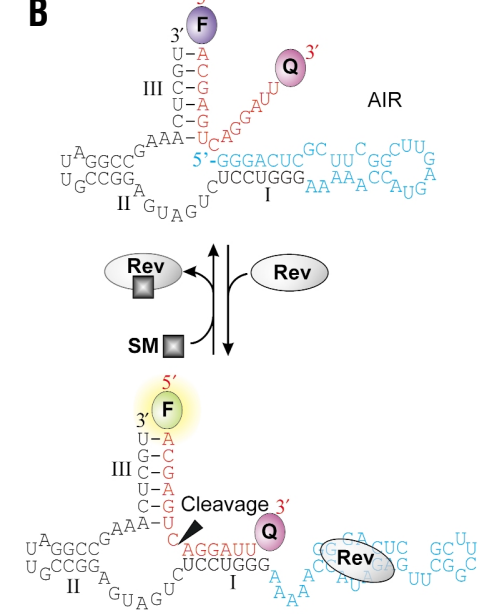

C
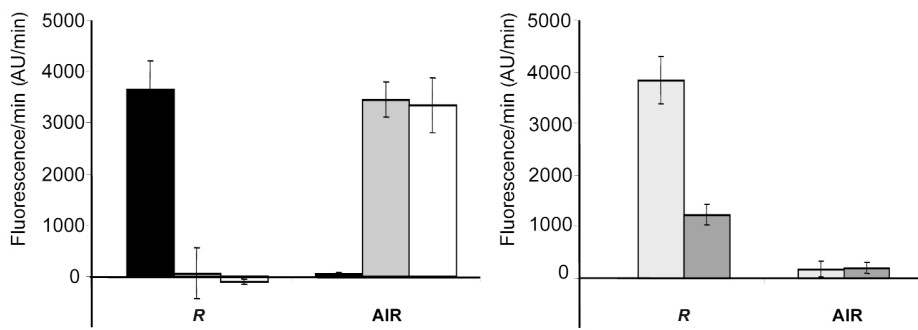

Figure 1. Rev-responsive and aptamer-inhibited ribozymes. (A) The Revresponsive ribozyme $(R)$ complexed with 13-mer substrate (red). The Revbinding element (cyan) was fused to helix II of the hammerhead ribozyme. In the presence of Rev peptide or protein, the $R$ is inhibited and no fluorescence is detected. Rev-binding small molecules (SM) displace the protein from its natural RNA-binding element and restore cleavage activity. $F$, fluorescein label (FAM); $\mathrm{Q}$, fluorescence quencher $N, N$-tetramethylrhodamine label (TAMRA). (B) In the aptamer-inhibited ribozyme (AIR), the anti-Rev aptamer (cyan) was attached via a penta-A-linker to the $5^{\prime}$ end of the hammerhead ribozyme. The aptamer domain should hybridize to the helix I substrate-binding domain, preventing the substrate (red) from complete annealing and cleavage. Rev induces a conformational shift in the aptamer, enabling hybridization and cleavage of the substrate and consequently generating a fluorescence signal. Rev-binding SM reverses this process and suppresses the fluorescence. (C) Inactivation of $R$ by $1 \mu \mathrm{M}$ Rev peptide or $5 \mu \mathrm{M}$ Rev protein and activation of AIR in the presence of $250 \mathrm{nM}$ Rev peptide or $1 \mu \mathrm{M}$ Rev protein. Black bar, ribozyme alone; gray bar, ribozyme + Rev peptide; white bar, ribozyme + Rev protein; AU, arbitrary units. (D) Reactivation of $R$ and reinhibition of AIR, respectively, by $100 \mu \mathrm{M}$ coumermycin $A_{1}$. Pale bar, ribozyme + Rev peptide + coumermycin $A_{1}$; dark bar, ribozyme + Rev protein + coumermycin $A_{1}$.

In the second approach, an anti-Rev-aptamer ${ }^{22}$ was fused to the $5^{\prime}$ end of the hammerhead ribozyme (Fig. 1B) to generate an aptamerinhibited ribozyme (AIR). In the absence of Rev, the aptamer domain hybridized to the hammerhead ribozyme to form a stem that prevented substrate annealing and, hence, cleavage. In the presence of the cognate protein or peptide, the aptamer instead formed a defined structure that rendered the hammerhead ribozyme's substrate-binding site accessible for annealing and cleavage of the external FRET-labeled substrate. Disruption of the aptamer-protein interaction by a small molecule allowed the original stem structure to form, again suppressing the generation of a fluorescence signal.

Screening for small molecules that interact with Rev. To test whether these assay formats were compatible with parallel screening formats, we searched for molecules that could disrupt the Rev-ribozyme complex. The 15-amino acid Rev ARM ${ }^{23-25}$ was mixed with the $R$ and AIR chimeric ribozymes shown in Figure 1A and $B$, respectively. A peptide concentration of $1 \mu \mathrm{M}$ was used in the $R$ reporter system, resulting in a 36-fold decrease in fluorescence intensity per time unit. The $250 \mathrm{nM}$ peptide concentration used in the AIR reporter system led to a 34 -fold increase in fluorescence intensity per time unit (Fig. 1C). In general, the stoichiometry of substrate cleavage can be easily related to the fluorescence per minute by determining the arbitrary units (AU) that correspond to complete cleavage of a given amount of substrate. For example, in Figure 1C each reaction contained $10 \mathrm{pmol}$ of the labeled substrate. Complete cleavage generated a fluorescence signal of $17,700 \mathrm{AU}$. Hence, the 3,600 AU/min observed for the initial activity of the noninhibited $R$ (Fig. 1C) corresponds to $2.0 \mathrm{pmol} / \mathrm{min}$ of cleaved substrate.

A model library of 96 structurally and functionally diverse antibiotics, each at a $100 \mu \mathrm{M}$ concentration, was then screened with each reporter system. This concentration was used because it permits observation of even weak effects of compounds in an initial evaluation of the library. Most of the library members generated signals within the threshold values that had been defined earlier ${ }^{18}$. However, three compounds were active in both formats: coumermycin $A_{1}$, nosiheptide, and patulin (data not shown and Supplementary Fig. 1 online; Fig. 1D). Control reactions with unmodified hammerhead ribozyme (HHR) not responsive to Rev were also carried out, and the signals observed with the two reporter ribozymes were divided by the signal obtained with the HHR. This procedure guarded against the identification of nonspecific effects on hammerhead cleavage activity $^{18}$. The disruption of the interaction between Rev and the reporter ribozymes occurred in a concentration-dependent manner, and median inhibitory concentration $\left(\mathrm{IC}_{50}\right)$ values of 7-15 $\mu \mathrm{M}$ were obtained for coumermycin and patulin (Fig. 2A). The results from both screening systems were in accord even though the readouts were reciprocal to one another. None of the active compounds affected the activity of the reporter ribozymes alone. The inhibition of the RNA-protein complex formation was independently confirmed by competition studies using a filter binding assay in which $5^{\prime}-{ }^{32} \mathrm{P}$-labeled reporter ribozymes complexed to Rev protein were eluted from nitrocellulose filters in the presence of increasing concentrations of coumermycin, patulin, or nosiheptide. Half-maximal inhibition values were of the same order as the values obtained by measuring the kinetics of substrate cleavage (Supplementary Fig. 2 online).

Identified compounds bind to Rev and not to RNA. To determine whether the identified compounds bound to the protein or the RNA, we carried out surface plasmon resonance (SPR) studies. Flow cells were derivatized with the reporter ribozymes, Rev peptide, or Rev protein. The active antibiotics bound to both Rev peptide and protein but not to RNA (Fig. 2B). We determined equilibrium dissociation constants of protein-antibiotic complexes by injecting increasing concentrations of antibiotics into flow cells derivatized with the full-length Rev protein (Fig. 2C). Fitting of the steady-state responses revealed a $K_{d}$ of $5.8 \mu \mathrm{M}$ for the coumermycin $A_{1}-$ Rev complex (Fig. 2D), and of $1.3 \mu \mathrm{M}$ for the patulin-Rev complex. No interaction was detected with representative members of the library that were negative in the screening assay (Fig. 2C). Binding specificity was assayed using other RNA-binding proteins from HIV-1, namely $\mathrm{Tat}^{26}$, which contains a semiconsensus arginine-rich motif that is found in several proteins, including the HIV-1 Rev protein ${ }^{27-29}$, and reverse transcriptase (HIV-1 RT). We also used the DNA-binding protein NF- $\kappa \mathrm{B}$ p52, and a protein with no known affinity to nucleic acids, cytohesin-1 (Table 1). This analysis revealed that coumermycin $A_{1}$ binds to cytohesin- 1 , NF- $\kappa B$, and HIV1 RT within an order of magnitude less tightly than to HIV-1 Rev. Coumermycin affinity to these proteins is 50-, 25-, and 12-fold 
A

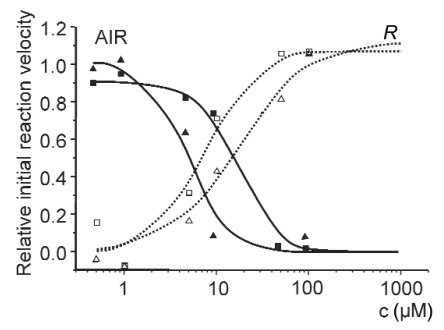

C

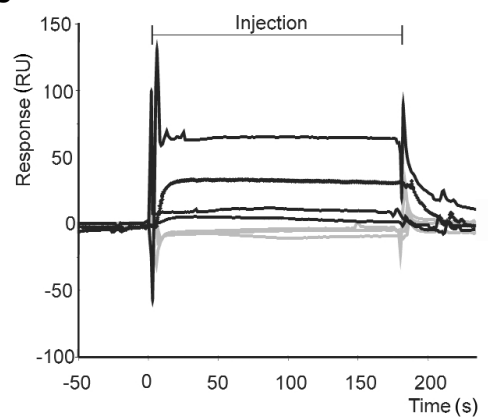

B

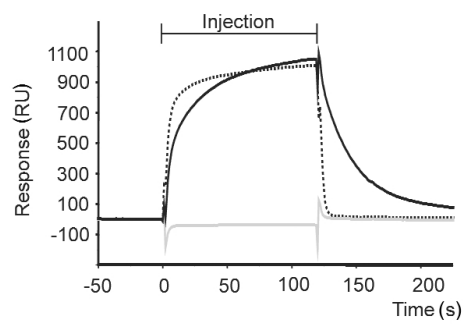

D

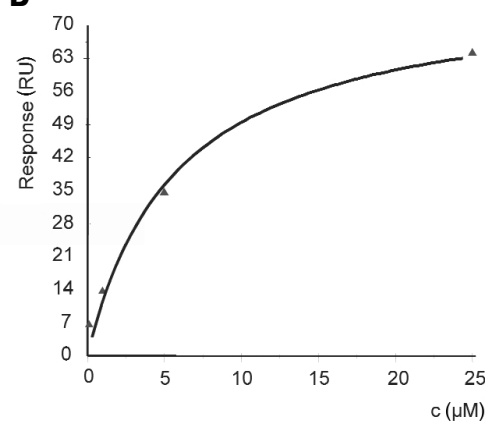

Figure 2. Interaction of Rev with small molecules. (A) Inactivation of the cleavage activity of AIR-Rev complex (straight lines) and activation of $R$-Rev complex (dotted lines) in the presence of increasing concentrations of patulin $(\boldsymbol{\Lambda}, \triangle)$ and coumermycin $A_{1}(\boldsymbol{\square}, \square)$. (B) SPR analysis of coumermycin $A_{1}$ binding to flow cells derivatized with Rev peptide (black dotted line), Rev protein (black straight line), and $R$ (gray line). No binding to $R$-derivatized surface was detectable. Binding to Rev peptide and Rev-derivatized surfaces was reversible. (C) Coumermycin $A_{1}$ binding to Rev-derivatized flow cell is concentration dependent. Coumermycin $A_{1}$ was injected at concentrations of $0.1 \mu \mathrm{M}$, $1 \mu \mathrm{M}, 5 \mu \mathrm{M}$, and $25 \mu \mathrm{M}$ (black lines). Rosamicin, griseovulvin, and streptolydigin are antibiotics identified as negative in the screen. Injection of $100 \mu \mathrm{M}$ of these compounds showed no response under the same SPR conditions (gray lines). (D) Curve fitting of response vs. concentration for coumermycin $A_{1}$ binding to immobilized Rev protein revealed a $K_{\mathrm{d}}$ of $5.8 \mu \mathrm{M}$.

reduced, respectively, as compared with the coumermycin-Rev affinity. Although Rev, among all tested proteins, clearly exhibited highest affinity to coumermycin, the HIV-1 Tat protein showed only a fourfold reduced affinity, indicating some degree of cross-affinity between the two related arginine-rich motifs of Tat and Rev. In contrast to coumermycin, patulin had the same affinity for all four nucleic acid-binding proteins, a pattern strongly indicative of a nonspecific binding mode. Further optimization of the assay thus must be directed toward decreasing the hit rate of nonspecific interactors.

An identified ligand inhibits HIV-1 replication. Coumermycin $A_{1}$ (Fig. 3A), the identified antibiotic that showed fairly specific binding to Rev, was then assayed for its ability to reduce HIV-1 replication in tissue culture cells. In parallel, antibiotic cytotoxicities were measured and compared with the cytotoxicity of the nonspecific protein interactor patulin. As shown in Figure 3B, HIV-1 replication was reduced in the presence of $5 \mu \mathrm{M}$ coumermycin $\mathrm{A}_{1}$ without any detectable cellular toxicity (Fig. 3C). In contrast, patulin exhibited a high level of toxicity at $5 \mu \mathrm{M}$, as might be expected for a nonspecific protein-binding drug (Fig. 3C). At 7.5 $\mu \mathrm{M}$ coumermycin $\mathrm{A}_{1}$ the effect was even more pronounced (Fig. 3B), but a low level of cellular toxicity was also observed (Fig. 3D). The differences between coumermycin and patulin with respect to specificity and toxicity illustrate the importance of a secondary evaluation of the "hits" resulting from the initial screening. On the other hand, these data show that our assays based on the Rev-responsive reporter ribozymes were able to identify quickly a previously unknown inhibitor of HIV-1 replication, and coumermycin may now serve as an interesting lead compound for the development of small molecules that are even more potent in reducing retrovirus replication.

Modular reporter ribozymes detect protein interactions. Given that protein-RNA and protein-small molecule interactions can be probed using our reporter ribozymes, it should also be possible to probe protein complexes and protein-protein interactions with the same system. To this end, we designed two additional reporter systems based on hammerhead and hairpin ribozymes ${ }^{30}$. These reporter ribozymes are referred to as the aptamer-hybridizing hairpin (AHP) and aptamerhybridizing hammerhead $(\mathrm{AHH})$ motifs. These reporter ribozymes contained sequences that were complementary to the anti-thrombin DNA aptamer, which binds to exosite 1 of human thrombin ${ }^{31}$ (Fig. 4A). In this configuration, added aptamers hybridized to and inactivated reporter ribozymes. Conversely, addition of a 20 - to 30 -fold excess of the cognate protein shifted the equilibrium in favor of the active aptamer structure and reactivated the ribozymes (Fig. 4A). Note that these reporter systems may be even more convenient than the direct conjugation of aptamers to ribozymes described above. Assay components such as the hybridizing aptamer and its protein target can be chemically modified or modularly swapped without altering the function of the underlying reporter ribozyme.

Aptamer-specific inhibition was shown for the thrombin-responsive hairpin ribozyme (AHP-Thr; Fig. 4B, columns 1 and 2) and hammerhead ribozyme (AHHThr; Supplementary Fig. 3 online, columns 1 and 2).

Only the anti-thrombin aptamer rendered the ribozyme inactive, whereas other DNA or RNA molecules containing unrelated nucleotide sequences did not function as inhibitors (data not shown). A 20- to 30-fold excess of $\alpha$-thrombin added to the aptamer-inhibited ribozymes led to $60-70 \%$ reactivation (column 4 ). Notably, $\gamma$-thrombin (which is identical to $\alpha$-thrombin but lacks exosite 1, the domain recognized by the aptamer) was unable to reactivate the ribozyme (column 3 ). In addition, the remarkable specificity of AHPThr for $\alpha$-thrombin is summarized in Figure 4C. Among a collection of 13 different proteins, $\alpha$-thrombin was able to reactivate AHP-Thr significantly, to a level of $60-70 \%$ of the non-aptamer-inhibited ribozyme activity. Human Factor Xa, the active form of the bloodclotting Factor X containing an exosite related to thrombin ${ }^{32}$, reactivated AHP-Thr only to a level of $10 \%$ of the noninhibited ribozyme. These results demonstrate that highly specific reporter ribozymes can be designed in a modular fashion and then generated in a straightforward manner. Table 1. Dissociation constants of compounds to Rev measured
by SPR

\begin{tabular}{lcc}
\hline \multirow{2}{*}{ Protein } & \multicolumn{2}{c}{ Dissociation constant $(\mu \mathbf{M})^{\mathrm{a}}$} \\
\cline { 2 - 3 } & Coumermycin $\mathbf{A}_{\mathbf{1}}$ & Patulin $^{\mathrm{a}}$ \\
\hline HIV-1 Rev & 5.8 & 1.3 \\
HIV-1 RT & 70.5 & 1.0 \\
HIV-1 Tat & 23.0 & 1.2 \\
NF-kB & 140 & 1.5 \\
Cytohesin-1 & 300 & - \\
\hline
\end{tabular}

${ }^{a} K_{d}$ values were determined in the same way as shown in Figure $2 \mathrm{C}$, D. Proteins used for comparison: NF- $\mathrm{KB}$ transcription factor $\mathrm{p} 52$; cytohesin-1, a cytoplasmic protein that acts as a guanine nucleotide exchange factor on small ras-like GTPases ${ }^{46,47}$. 
A

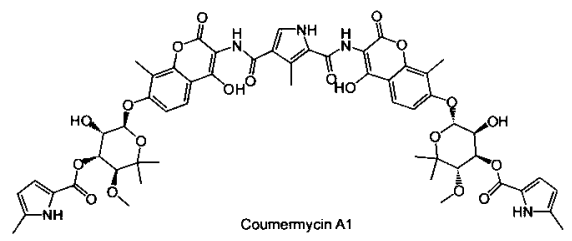

B

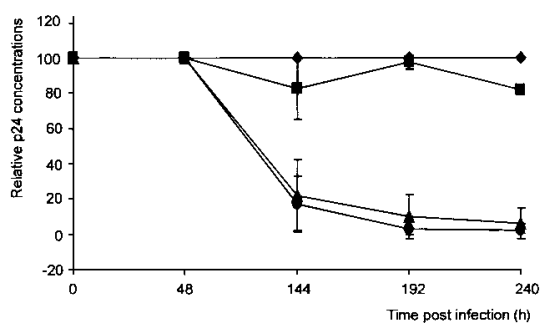

C

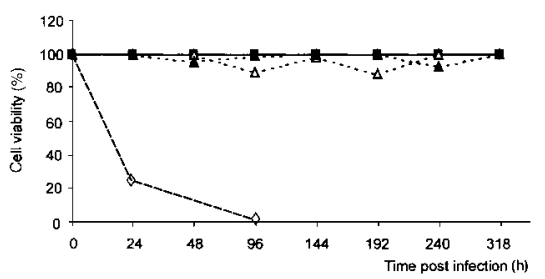

D

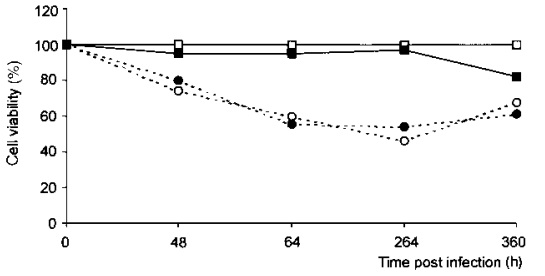

Figure 3. Coumermycin $A_{1}$ inhibits HIV-1 replication. (A) Structure of coumermycin $A_{1}$. (B) Effect of coumermycin $A_{1}$ on HIV-1 p24 levels. H9 cells infected with HIV-1 $1_{\text {III }}$ were grown in the presence of $1 \mu \mathrm{M}(\mathbf{\square}), 5 \mu \mathrm{M}$ $(\boldsymbol{\Delta}), 7.5 \mu \mathrm{M}(\bullet)$, or no coumermycin $\mathrm{A}_{1}(\mathbf{)})$. p24 concentrations are shown relative to infected cells treated with similar volumes of dimethyl sulfoxide alone ( $)$ to adjust for variations in assay reagents and between assay days. All data represent the average of two replicates of the experiments on different days. (C) Toxicity of coumermycin and patulin. Effects of $5 \mu \mathrm{M}$ coumermycin $\mathrm{A}_{1}(\boldsymbol{\Delta}$ and $\triangle$ ) on H9 cells in comparison to $5 \mu \mathrm{M}$ patulin $(\diamond)$. The viability of infected cells (closed symbols) and noninfected cells (open symbols) in the presence of compounds was determined relative to cell controls ( $\square$ and $\square$ ). (D) Same experiment in presence of $7.5 \mu \mathrm{M}$ coumermycin $A_{1}(\circ$ and $\bullet)$.

To test whether the ribozyme screens could potentially detect protein-protein interactions, we carried out a similar experiment in the presence of increasing concentrations of the exosite 1-binding thrombin inhibitor hirudin ${ }^{33}$. As shown in Figure 4B (columns 5-9), AHP-Thr sensitively reported the $\alpha$-thrombin-hirudin proteinprotein interaction in a concentration-dependent manner. Analogous data were obtained with AHH-Thr (Supplementary Fig. 3 online). Moreover, a sulfated version of the exosite 1-specific C-terminal 13-mer peptide of hirudin has been shown to bind thrombin with greater affinity than the nonsulfated version of this fragment ${ }^{34}$, and a roughly threefold difference in the $\mathrm{IC}_{50}$ values can be detected by AHH-Thr (Supplementary Fig. 4 online). The sulfated peptide suppressed the fluorescence signal with an $\mathrm{IC}_{50}$ of $1.8 \mu \mathrm{M}$ compared with $6 \mu \mathrm{M}$ for the nonsulfated version. The thrombin-responsive reporter ribozymes can potentially be applied in high-throughput screens for small molecules or peptides that function as superior thrombin inhibitors. Notably, the protein antithrombin III (AT III), which specifically binds to exosite 2 of $\alpha$-thrombin with high affinity, was unable to disrupt the aptamer-thrombin complex and left both reporter ribozymes active (Fig. 4B, column 10; see also
Supplementary Fig. 3 online). Thus, advance knowledge of the target epitope recognized by an aptamer should permit immediate identification of the domains involved in newly discovered protein-protein interactions, provided that aptamer release results from binding competition rather than from indirect effects.

Conclusions. We have shown that protein-responsive reporter ribozymes can signal the presence of a cognate protein, and can be up- or downregulated by compounds of high or low molecular mass that compete with regulatory oligonucleotides for protein binding. The ribozyme reporters described here can thus be used to identify directly and measure novel molecular interactions in automated FRET- or fluorescence-dequenching assays. That aptamers can be generated for a large variety of targets ${ }^{35}$ using automated selection methods ${ }^{36}$ suggests that these methods should be compatible with the parallel screening of large compound or protein libraries. Moreover, the ribozyme-based assays can be used with minimal modifications compared with existing assay formats, as they do not require labeling of compounds or proteins, and use only small amounts of analytes because positive signals are catalytically amplified. Although other examples of nucleoprotein ribozymes have been described $^{37-39}$, it is unclear to what extent they can be modularly adapted to multiple high-throughput assay formats.

Furthermore, the finding that ribozymes can be up- or downregulated by the interplay between interacting oligonucleotides, proteins, peptides, and even small molecules provides a synthetic model for the evolution of complex cellular machineries like the ribosome or the spliceosome that are thought to have been derived from RNA catalysts ${ }^{40,41}$. According to the RNA-world hypothesis, proteins that bound nonspecifically to functional RNAs may have represented an early step in the evolution of large natural RNA-protein machineries ${ }^{42}$. It is plausible that small oligonucleotides may have participated in the regulation of early RNA catalysts through nonspecific or specific interactions in trans. Previous work has shown that ribozymes can sense multiple regulatory analytes in parallel ${ }^{38,43,44}$. Here we provide experimental evidence that ribozyme activity, controlled by a regulatory protein, can also be reverted in the presence of a second interaction partner of high or low molecular mass that complexes the regulatory protein. In the RNA world, effector molecules could have interacted with specific regulatory RNAs to modulate ribozyme function and may have had functions similar to those of second messengers and allosteric effectors in modern biochemistry. As the RNA world evolved, the regulatory RNAs may have eventually served as adapters that allowed the chemistries of effector molecules or effector complexes to augment the nascent catalytic abilities of ribozymes. In this sense, our system may represent a simple chemical model for early steps in the evolution of the complex RNA-protein machines that ultimately derived from catalytic RNA.

\section{Experimental protocol}

Design and synthesis of Rev-dependent reporter ribozymes. Reporter ribozymes were synthesized by PCR amplification and in vitro transcription using the following DNA templates: $R, 5^{\prime}$-TCTAATACGACTCACTATAGGGTCCTCTGATGAGCTCTGGGCGCAGCTTCGGCTGACGGTACTTGCGAAACTCGT-3'; AIR, 5'-TCTAATACGACTCACTATAGGGACTCGCTTCGGCTTGAGATACCAAAAAGGGTCCTCTGATGAGGCCGTTAGGCCGAAACTCGT-3'; hammerhead wild-type control HHR, 5'TCTAATACGACTCACTATAGGGTCCTCTGATGAGGCCGTTAGGCCGAAACTCGT-3'; FRET-labeled RNA substrate, 5'-Fam-ACGAGUCAGGAUU-Tamra-3'; Rev peptide sequence, TRQARRNRRRRWRERQR.

Ribozyme reactions. Multiple-turnover cleavage reactions ( $50 \mu \mathrm{l})$ were carried out in $\mathrm{HHR}$ buffer $(50 \mathrm{mM}$ Tris- $\mathrm{HCl}, \mathrm{pH} 7.9,25 \mathrm{mM} \mathrm{NaCl})$ at $32^{\circ} \mathrm{C}$ in 96 -well plates. Ribozyme $(10 \mathrm{nM})$ and FRET-labeled substrate $(200 \mathrm{nM})$ were preincubated in HHR buffer $\left(25^{\circ} \mathrm{C}, 5 \mathrm{~min}\right)$, followed by optional addition of Rev and/or antibiotic. After incubation $\left(32^{\circ} \mathrm{C}, 5 \mathrm{~min}\right)$, reactions were started by addition of $\mathrm{MgCl}_{2}$ to a final concentration of $8 \mathrm{mM}$. Negative controls without ribozymes were routinely included and automatically subtracted. 
A

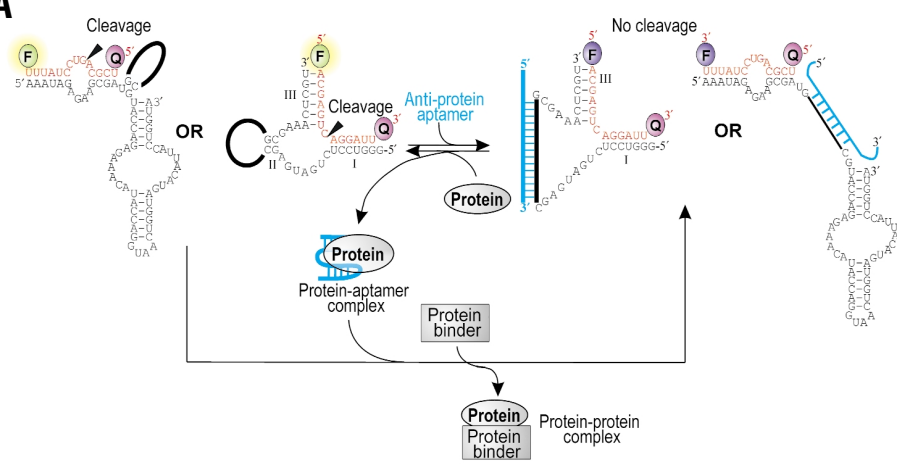

B

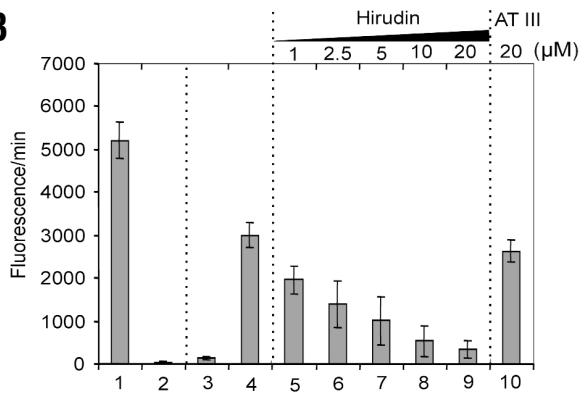

C

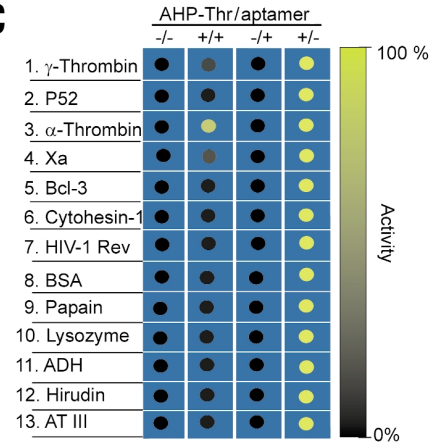

Figure 4. Specific protein-protein interactions as a function of cleavage activities for AHH-Thr and AHP-Thr. (A) Scheme for anti-protein aptamer-regulated hammerhead and hairpin ribozymes ${ }^{45}$ and their response to aptamer-specific proteins. A specific protein-protein interaction should decoy the target protein from the aptamer (cyan), which can then hybridize to an antisense sequence (bold lines) within a ribozyme, rendering the ribozyme inactive for substrate (red) cleavage. F, Fluorescein label (FAM); Q, fluorescence quencher (TAMRA). (B) Protein-protein interactions of $\alpha$-thrombin and hirudin can be detected in a domain-specific fashion with AHP-Thr (50 nM). 1, Ribozyme alone;

2, ribozyme/anti- $\alpha$-thrombin-aptamer (1:1). Equimolar amounts of aptamer resulted in complete inhibition of AHP-Thr. 3, Ribozyme/anti-thrombin aptamer/ $\gamma$-thrombin (1:1:20). $\gamma$-Thrombin lacking exosite 1 of thrombin was unable to decoy the aptamer from AHP-Thr. 4, Ribozyme/anti-thrombin aptamer/ $\alpha$-thrombin (1:1:20). $60 \%$ of AHP-Thr activity was restored. $5-9$, Same as 4 with increasing concentrations of hirudin $(1-20 \mu \mathrm{M})$. At increasing concentrations, hirudin competed with the aptamer for binding to exosite 1 of $\alpha$-thrombin, resulting in aptamer release and inhibition of ribozymes. 10, Same as 4 with $20 \mu \mathrm{M}$ of exosite 2 -specific antithrombin III (AT III). AT III binding to exosite 2 of $\alpha$-thrombin showed no effect. The AHH-Thr ribozyme (30 nM) yielded the same results as AHP-Thr (supporting data S3). In both systems (AHH-Thr and AHP-Thr), neither $\alpha$ - and $\gamma$-thrombin nor hirudin or AT III had an effect on ribozyme alone or ribozyme + aptamer. (C) Reporter ribozyme AHP-Thr specifically reported the interaction of the anti-thrombin aptamer with the exosite 1 of $\alpha$-thrombin. Among a set of randomly selected control proteins, only $\alpha$-thrombin activated the aptamerinhibited AHP-Thr. None of the 13 proteins affected the activity of the ribozyme in the absence of the aptamer. 1, Human $\gamma$-thrombin; 2, NF-kB transcription factor $\mathrm{p} 52 ; 3$, human $\alpha$-thrombin; 4 , human Factor $\mathrm{Xa} ; 5, \mathrm{Bcl}-3$, a member of the $\mathrm{I \kappa B}$ protein family; 6 , cytohesin-1, a cytoplasmic regulatory protein with guanine nucleotide exchange factor function; 7, Rev protein of HIV-1; 8, BSA; 9 , papain; 10 , hen egg white lysozyme; $11, A D H$, alcohol dehydrogenase; 12 , hirudin; 13 , antithrombin III.
Screening the antibiotic library for Rev interactors. Screens were done on 96-well plates. For each antibiotic, three different reactions were carried out containing hammerhead, reporter, or no ribozyme, antibiotic $(100 \mu \mathrm{M})$, Rev peptide ( $1 \mu \mathrm{M}$ for $R, 250 \mathrm{nM}$ for AIR), and substrate ( $200 \mathrm{nM}$ ). Each plate also contained standard ribozyme reactions (HHR, $1 \mu \mathrm{M} R$, or $250 \mathrm{nM}$ AIR) without antibiotic as a positive control. The "no ribozyme" negative control was subtracted from the values obtained in the presence of ribozymes. From these values, the initial reaction velocity (which correlated with the fluorescence increase) within the first 5 min was determined. The resulting values for $R$ and AIR were divided by the value obtained for HHR.

Surface plasmon resonance measurements. SPR measurements were done in a Biacore 3000 (Biacore AB, Uppsala, Sweden) using automated procedures. Flow cells contained 1,800 RU Rev peptide, 5,000 RU of protein, and 1,600 RU of RNA. For $K_{\mathrm{d}}$ determinations, cells derivatized with $500 \mathrm{RU}$ Rev were used. All binding experiments were carried out in HHR buffer at $25^{\circ} \mathrm{C}$ by injection of $20-30 \mu \mathrm{l}$ sample solution at a flow rate of $10 \mu \mathrm{l} / \mathrm{min}$ and with in-line reference subtraction of underivatized surfaces. Fitting of obtained binding sensograms was carried out with the Biacore evaluation software.

HIV-1 replication assays. Cells were grown at $37^{\circ} \mathrm{C}$ in a $5 \% \mathrm{CO}_{2}$ atmosphere in RPMI-1640 medium supplemented with $10 \%$ FCS, $10 \mathrm{mM}$ HEPES, $1 \mathrm{mM}$ sodium pyruvate, $1.5 \mathrm{~g} / \mathrm{ml} \mathrm{NaHCO}_{3}, 2 \mathrm{mM}$ L-glutamine, $2.5 \mathrm{U} / \mathrm{ml}$ penicillin, and $2.5 \mathrm{mg} / \mathrm{ml}$ streptomycin. For infection assays, $1.2 \times$ $10^{7} \mathrm{H} 9$ cells were washed in sterile PBS and pelleted by spinning at 1,500 rpm $(430 \mathrm{~g})$ for $5 \mathrm{~min}$. Half of the cells were resuspended in $3 \mathrm{ml}$ of HIV$1_{\text {III }}$ in R10 with a p24 concentration of $4 \mathrm{ng} / \mathrm{ml}$ and incubated for $1 \mathrm{~h}$ with agitation every $10 \mathrm{~min}$ to redisperse cells. A parallel mock infection was carried out in which the other half of the cells were incubated in $3 \mathrm{ml}$ of R10 only. Cells were washed and pelleted as before and resuspended in fresh R10. Infected cells were divided into three $25 \mathrm{~cm}^{2}$ flasks in a total volume of $7.5 \mathrm{ml} \mathrm{R} 10$ supplemented with $0 \mu \mathrm{M}, 1 \mu \mathrm{M}, 5 \mu \mathrm{M}$, or $7.5 \mu \mathrm{M}$ of coumermycin. Mock-infected cells were treated likewise. A $1.5 \mathrm{ml}$ sample of each cell culture was taken approximately every two days, counted for cell viability, and assayed using a p24 antigen ELISA kit. The total volume of each culture was maintained by adding $1.5 \mathrm{ml}$ fresh R10 supplemented with the appropriate concentration of coumermycin.

Thrombin-dependent ribozyme assays. AHH-Thr (30 nM) or AHP-Thr $(50 \mathrm{nM})$ were preincubated for $5 \mathrm{~min}$ at $32^{\circ} \mathrm{C}$ in HHR buffer or hairpin (HP) buffer (50 mM Tris-HCl, pH 7.5) with $200 \mathrm{nM}$ HHR substrate or $1 \mu \mathrm{M}$ HP substrate (5'-Tamra-UCGCAGUCCUAUUU-Fam-3'). Effectors (50 nM anti-thrombin DNA aptamer 5'-GGTTGGTGTGGTTGG-3', $1 \mu \mathrm{M}$ proteins) were then added and the reaction was further incubated for $10 \mathrm{~min}$ at $32^{\circ} \mathrm{C}$. The reaction was initiated by adding $\mathrm{MgCl}_{2}$ to a final concentration of $8 \mathrm{mM}$ (AHH-Thr) or $20 \mathrm{mM}$ (AHP-Thr). The initial fluorescence/min values were corrected by subtracting values derived from reactions lacking ribozyme.

Note: Supplementary information is available on the Nature Biotechnology website.

\section{Acknowledgments}

This work was supported by the Deutsche Forschungsgemeinschaft (to M.F.) and by National Institutes of Health grants AI-36083 and GM-61789 (to A.D.E.). We thank Julian Davies (University of Vancouver) for providing the antibiotics library, Christoph Müller (European Molecular Biology Laboratory, Grenoble) for NFאB, p52, and Bcl-3, Tobias Restle (MaxPlanck-Insititut für Molekulare Physiologie, Dortmund) for HIV-RT, Günter Mayer for helpful discussions, and Michael Hoch and Waldemar Kolanus (University of Bonn) for comments on the manuscript. The following reagents were obtained through the National Institutes of Health AIDS Research and Reference Reagent Program (National Institute of Allergy and Infectious Diseases): HIV-1 Tat protein from John Brady and $\mathrm{H} 9$ cells and

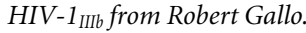

\section{Competing interests statement}

The authors declare competing financial interests: see the Nature Biotechnology website (http://biotech.nature.com) for details. 
1. Thompson, L. \& Ellman, J. Synthesis and applications of small molecule libraries. Chem. Rev. 96, 555-600 (1996).

2. Jayawickreme, C.K. \& Kost, T.A. Gene expression systems in the development of high-throughput screens. Curr. Opin. Biotechnol. 8, 629-634 (1997).

3. Rademann, J. \& Jung, G. Drug discovery. Integrating combinatorial synthesis and bioassays. Science 287, 1947-1948 (1998).

4. Mendelsohn, A.R. \& Brent, R. Protein interaction methods-toward an endgame. Science 284, 1948-1950 (1999).

5. Pandey, A. \& Mann, M. Proteomics to study genes and genomes. Nature 405 , 837-846 (2000).

6. Phizicky, E.M. \& Fields, S. Protein-protein interactions: methods for detection and analysis. Microbiol. Rev. 59, 94-123 (1995).

7. Cech, T.R. in The RNA World (eds Gesteland, R.F. \& Atkins, J.F.) 239-269 (Cold Spring Harbor Laboratory Press, Cold Spring Harbor, New York, 1993).

8. Warashina, M., Takagi, Y., Stec, W. J. \& Taira, K. Differences among mechanisms of ribozyme-catalyzed reactions. Curr. Opin. Biotechnol. 11, 354-362 (2000).

9. Long, D.M. \& Uhlenbeck, O.C. Self-cleaving catalytic RNA. FASEB J. 7, 25-30 (1993).

10. Doherty, E.A. \& Doudna, J.A. Ribozyme structures and mechanisms. Annu. Rev. Biochem. 69, 597-615 (2000)

11. Walter, N.G. \& Burke, J.M. The hairpin ribozyme: structure, assembly and catalysis. Curr. Opin. Chem. Biol. 2, 24-30 (1998).

12. Verma, S., Vaish, N.K. \& Eckstein, F. Structure-function studies of the hammerhead ribozyme. Curr. Opin. Chem. Biol. 1, 532-536 (1997).

13. Rossi, J.J. Ribozymes, genomics and therapeutics. Chem. Biol. 6, R33-R37 (1999).

14. Bramlage, B., Luzi, E. \& Eckstein, F. Designing ribozymes for the inhibition of gene expression. Trends Biotechnol. 16, 434-438 (1998).

15. Sullenger, B.A. \& Cech, T.R. Ribozyme-mediated repair of defective mRNA by targeted, trans-splicing. Nature 371, 619-622 (1994).

16. Sullenger, B.A. Revising messages traveling along the cellular information superhighway. Chem. Biol. 2, 249-253 (1995).

17. Jenne, A., Gmelin, W., Raffler, N. \& Famulok, M. Real-time characterization of ribozymes by fluorescence resonance energy transfer (FRET). Angew. Chem. Int. Ed. 38, 1300-1303 (1999).

18. Jenne, A. et al. Rapid identification and characterization of hammerheadribozyme inhibitors using fluorescence-based technology. Nat. Biotechnol. 19, 56-61 (2001).

19. Soukup, G.A. \& Breaker, R.R. Engineering precision RNA molecular switches. Proc. Natl. Acad. Sci. USA 96, 3584-3589 (1999).

20. Peterson, R.D. \& Feigon, J. Structural change in Rev responsive element RNA of HIV-1 on binding Rev peptide. J. Mol. Biol. 264, 863-877 (1996).

21. Gosser, Y. et al. Peptide-triggered conformational switch in HIV-1 RRE RNA complexes. Nat. Struct. Biol. 8, 146-150 (2001).

22. Giver, L. et al. Selective optimization of the Rev-binding element of HIV-1. Nucleic Acids Res. 21, 5509-5516 (1993)

23. Battiste, J.L. et al. Alpha helix-RNA major groove recognition in an HIV-1 rev peptide-RRE RNA complex. Science 273, 1547-1551 (1996).

24. Hung, L.W., Holbrook, E.L. \& Holbrook, S.R. The crystal structure of the Rev binding element of HIV-1 reveals novel base pairing and conformational variability. Proc. Natl. Acad. Sci. USA 97, 5107-5112 (2000).

25. Ippolito, J.A. \& Steitz, T.A. The structure of the HIV-1 RRE high affinity rev binding site at 1.6 Å resolution. J. Mol. Biol. 295, 711-717 (2000).

26. Bohan, C.A et al. Analysis of Tat transactivation of human immunodeficiency virus transcription in vitro. Gene Expr. 2, 391-407 (1992).

27. Burd, C.G. \& Dreyfuss, G. Conserved structures and diversity of functions of RNAbinding proteins. Science 265, 615-621 (1994).

28. Mattaj, I.W. RNA recognition: a family matter? Cell 73, 837-840 (1993)

29. Patel, D.J. Adaptive recognition in RNA complexes with peptides and protein modules. Curr. Opin. Struct. Biol. 9, 74-87 (1999).

30. Esteban, J.A., Banerjee, A.R. \& Burke, J.M. Kinetic mechanism of the hairpin ribozyme. Identification and characterization of two nonexchangeable conformations. J. Biol. Chem. 272, 13629-13639 (1997).

31. Bock, L.C., Griffin, L.C., Latham, J.A., Vermaas, E.H. \& Toole, J.J. Selection of single-stranded DNA molecules that bind and inhibit human thrombin. Nature 355 564-566 (1992).

32. Rezaie, A.R. Heparin-binding exosite of factor Xa. Trends Cardiovasc. Med. 10, 333-338 (2000)

33. Rydel, T.J. et al. The structure of a complex of recombinant hirudin and human $\alpha$ thrombin. Science 249, 277-280 (1990).

34. Niehrs, C., Huttner, W.B., Carvallo, D. \& Degryse, E. Conversion of recombinant hirudin to the natural form by in vitro tyrosine sulfation. Differential substrate specificities of leech and bovine tyrosylprotein sulfotransferases. J. Biol. Chem. 265, 9314-9318 (1990).

35. Famulok, M., Blind, M. \& Mayer, G. Intramers as promising new tools in functional proteomics. Chem. Biol. 8, 931-939 (2001).

36. Cox, J.C. \& Ellington, A.D. Automated selection of anti-protein aptamers. Bioorg. Med. Chem. 9, 2525-2531 (2001).

37. Herschlag, D., Khosla, M., Tsuchihashi, Z. \& Karpel, R.L. An RNA chaperone activity of non-specific RNA binding proteins in hammerhead ribozyme catalysis. EMBO J. 13, 2913-2924 (1994).

38. Robertson, M.P. \& Ellington, A.D. In vitro selection of nucleoprotein enzymes. Nat. Biotechnol. 19, 650-655 (2001)

39. Atsumi, S., Ikawa, Y., Shiraishi, H. \& Inoue, T. Design and development of a catalytic ribonucleoprotein. EMBO J. 20, 5453-5460 (2001).

40. Nissen, P., Hansen, J., Ban, N., Moore, P.B. \& Steitz, T.A. The structural basis of ribosome activity in peptide bond synthesis. Science 289, 920-930 (2000).

41. Valadkhan, S. \& Manley, J.L. Splicing-related catalysis by protein-free snRNAs. Nature 413, 701-707 (2001).

42. Herschlag, D. RNA chaperones and the RNA folding problem. J. Biol. Chem. 270 , 20871-20874 (1995)

43. Robertson, M.P. \& Ellington, A.D. Design and optimization of effector-activated ribozyme ligases. Nucleic Acids Res. 28, 1751-1759 (2000).

44. Jose, A.M., Soukup, G.A. \& Breaker, R.R. Cooperative binding of effectors by an allosteric ribozyme. Nucleic Acids Res. 29, 1631-1637 (2001).

45. Vitiello, D., Pecchia, D.B. \& Burke, J.M. Intracellular ribozyme-catalyzed transcleavage of RNA monitored by fluorescence resonance energy transfer. RNA 6, 628-637 (2000)

46. Geiger, $C$. et al. Cytohesin-1 regulates $\beta$-2 integrin-mediated adhesion through both ARF-GEF function and interaction with LFA-1. EMBO J. 19, 2525-2536 (2000).

47. Mayer, G. et al. Controlling small guanine-nucleotide-exchange factor function through cytoplasmic RNA intramers. Proc. Natl. Acad. Sci. USA 98, 4961-4965 (2001). 